\title{
Irradiation effects on antibody performance in the frame of biochip-based instruments development for space exploration
}

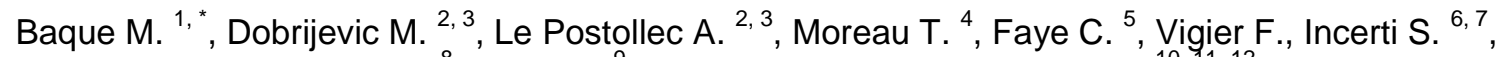 \\ Coussot G. ${ }^{8}$, Caron J. ${ }^{9}$, Vandenabeele Trambouze Odile ${ }^{10,11,12}$
}

${ }^{1}$ Univ Roma Tor Vergata, Dept Biol, Rome, Italy.

${ }^{2}$ Univ Bordeaux, LAB, UMR 5804, F-33270 Florac, France.

${ }^{3}$ CNRS, LAB, UMR 5804, F-33270 Florac, France.

${ }^{4}$ Univ Clermont Ferrand, INSERM U1103, GReD, CNRS UMR6293, Aubiere, France.

${ }^{5}$ Cap Alpha, COLCOM, F-34830 Clapiers, France.

${ }^{6}$ Univ Bordeaux, CENBG, UMR 5797, F-33170 Gradignan, France.

${ }^{7}$ CNRS, IN2P3, CENBG, UMR 5797, F-33170 Gradignan, France.

${ }^{8}$ Univ Montpellier, Inst Biomol Max Mousseron, Fac Pharm, CNRS,Unite Mixte Rech 5247, F-34093

Montpellier 5, France.

${ }^{9}$ Inst Bergonie, Dept Radiotherapy, Ctr Comprehens Canc, F-33076 Bordeaux, France.

${ }_{11}^{10}$ Univ Bretagne Occidentale, LMEE, UEB, IUEM UMR 6197, Plouzane, France.

${ }^{11}$ CNRS, IUEM UMR 6197, LMEE, Plouzane, France.

12 IFREMER, UMR6197, LMEE, Plouzane, France.

*Corresponding author : M. Baqué, email address : mickael.baque@gmail.com

\begin{abstract}
:
Several instruments based on immunoassay techniques have been proposed for life-detection experiments in the framework of planetary exploration but few experiments have been conducted so far to test the resistance of antibodies against cosmic ray particles. We present several irradiation experiments carried out on both grafted and free antibodies for different types of incident particles (protons, neutrons, electrons and C-12) at different energies (between $9 \mathrm{MeV}$ and $50 \mathrm{MeV}$ ) and different fluences. No loss of antibodies activity was detected for the whole set of experiments except when considering protons with energy between 20 and $30 \mathrm{MeV}$ (on free and grafted antibodies) and fluences much greater than expected for a typical planetary mission to Mars for instance. Our results on grafted antibodies suggest that biochip-based instruments must be carefully designed according to the expected radiation environment for a given mission. In particular, a surface density of antibodies much larger than the expected proton fluence would prevent significant loss of antibodies activity and thus assuring a successful detection.
\end{abstract}

Keywords : astrobiology, biochip, cosmic rays, search for extraterrestrial life 


\section{1. Introduction}

52 Among the next tools to search for signs of past or present life in our Solar System, 53 several instruments based on the biochip technology have been proposed in the 54 framework of planetary exploration. A biochip is a miniaturized device composed of 55 molecular recognition tools (or affinity receptors) like antibodies (Baqué et al. 2011b;

56 de Diego-Castilla et al. 2011; Parro et al. 2005, 2011a; Sims et al. 2005, 2012) or 57 aptamers (Baqué et al. 2011a), which allows the detection of hundreds of different 58 compounds in a single assay. Widely developed for biotechnology use and medical or 59 environmental diagnostics (see for example Wang 2006), miniaturized instruments

60 based on biochips have been indeed proposed and studied for biosignature detection in 61 an astrobiological context since more than 15 years (McKay et al. 2000; Parro et al. 62 2005; Le Postollec et al. 2007; Sims et al. 2005).

63 Mars, one of the most probable planetary body where to find signs of extinct or extant

64 life outside of Earth, is the target of many upcoming dedicated missions: ESA-

65 Roscosmos' ExoMars rover in 2016-2018, NASA's Mars2020 rover (a follow-up of to

66 the Curiosity rover) and the Icebreaker mission concept proposed for a 2021 launch to

67 be part of NASA's Discovery program (McKay et al., 2013). Different space

68 instruments based on the biochip technology and using antibodies have been proposed

69 for these future missions: the Life Marker Chip (LMC) (Martins 2011; Sephton et al.

70 2013; Sims et al. 2012), and the Signs Of LIfe Detector (SOLID) (Parro et al. 2005,

$712008,2011 a, 2011 b)$. Another project, the Biochip for Organic Matter Analysis in

72 Space (BiOMAS), proposes to combine both antibodies and aptamers in a single 73 instrument (Baqué et al. 2011b, 2011a; Le Postollec et al. 2007). Recently, first in the

74 framework of Mars2020 announcement of opportunity, and then in the framework of

75 NASA's Discovery 2014 announcement of opportunity, these different teams have 
76 united to work on the SOLID instrument proposal for the Icebreaker mission and thus to

77 contribute with their different expertise to improve the technological readiness level of a

78 biochip-based instrument for space exploration (Manchado et al. 2015; McKay et al.

79 2013; Smith \& Parro 2014).

80 Indeed, although biochips are known to be very sensitive tools to detect specific target

81 molecules, their sensitivity is related to the presence of functional affinity receptors. In

82 order to develop a "space biochip", it is thus necessary to ensure that these biological

83 receptors will survive space hazards. In particular, due to the very sparse data on this

84 topic, it is important to determine the behavior of these biological receptors under

85 cosmic particles irradiation.

86 Le Postollec et al. (2009a) performed simulations with the Geant4 Monte Carlo toolkit

87 in order to estimate the radiation environment that a biochip would face if it were placed

88 into a rover dedicated to explore Mars' surface. Ionizing doses accumulated and fluxes

89 of particles entering the biochip have been established for both the Earth-Mars transit

90 and the journey on Mars' surface. Neutrons and gammas appear as dominant radiation

91 species on Mars' soil whereas protons dominate during the interplanetary travel. These

92 results have been confirmed by other studies done by McKenna-Lawlor et al. (2012)

93 and Derveni et al. (2012). Moreover, these simulations can today be confronted to the

94 real radiation environment of an actual mission to Mars as it was monitored by the

95 Radiation Assessment Detector (RAD) instrument on-board the Mars science laboratory

96 spacecraft on cruise to Mars and continue to be recorded by the rover Curiosity directly

97 on its surface (Hassler et al. 2013; Kim et al. 2014; Zeitlin et al. 2013). Indeed, the total

98 cosmic radiation dose rate of $210 \pm 40 \mu \mathrm{Gy} /$ day (Hassler et al. 2013) recorded at Gale

99 Crater by Curiosity and the one measured inside the Mars Science Laboratory

100 spacecraft during its cruise to Mars (481 $\pm 80 \mu \mathrm{Gy} /$ day) (Zeitlin et al. 2013) proved to 
101 be in the same order of magnitude as model predictions with respectively $\sim 840 \mu \mathrm{Gy} / \mathrm{day}$

102 (without any shielding) for the Martian surface and $240 \mu \mathrm{Gy} / \mathrm{day}$ for the Earth-Mars

103 transit considering only GCR (galactic cosmic rays) contribution (Le Postollec et al.

104 2009a).

105

106 Considering the lack of experimental data about cosmic rays effect on antibodies,

107 particularly under lyophilized (freeze-dried) state, our team decided to investigate the

108 effects of different types of particles at several energies. Our objective is first to study

109 and measure cosmic rays effects on biological receptors and second to define well-

110 adapted protections for a biochip-based instrument if we find evidences that cosmic rays

111 might have deleterious effect on their performances. In a first study, Le Postollec et al.

112 (2009b) performed neutrons irradiation on both antibodies and fluorescein dyes (used

113 for detection of recognition events) at two energies ( 0.6 and $6 \mathrm{MeV})$ and with different

114 fluences. Sample analyses demonstrated that, in tested conditions, neutrons do not affect

115 antibody recognition capability and fluorescence dye intensity. More recently, the

116 effects of $2 \mathrm{MeV}$ protons on antibody performances (Baqué et al. 2011b) were

117 investigated. These studies showed that this irradiation process did not affect the

118 performances of antibodies as molecular recognition tools. In addition, printed antibody

119 and Alexa-647 fluorescent dye were demonstrated to be stable between 1.18 and 1.33

120 MeV gamma radiation (de Diego-Castilla et al. 2011). Finally, Derveni et al. (2012)

121 tested five antibodies freeze-dried in a variety of protective molecular matrices and 122 exposed to $50 \mathrm{MeV}$ protons. They showed that at a representative Mars-mission-dose,

123 none of the antibodies studied exhibited any evidence of activity loss due to the 124 radiation. 
125 In the present paper, we broaden these previous studies to test the effect of electrons,

126 carbon ions, protons (at different energies) and neutrons (at higher energies) on the

127 recognition capability of antibodies (summarized in Fig 1). As protons and neutrons

128 dominate the radiation environment during the Earth-Mars transit and on the Martian

129 surface, we tested different high-energy particles from 15 to $50 \mathrm{MeV}$ at different

130 fluences. Moreover, other damaging particles are significantly present in cosmic and

131 solar radiations such as carbon ions and electrons.

132 Chemicals and biological materials used to perform the experiments are given in section

133 2. Section 3 describes samples preparation, particles irradiation parameters and analysis

134 protocols. Results are presented in section 4. The last section draws conclusions on this 135 study.

\section{2. Material}

138 Monoclonal anti-Horseradish Peroxydase antibodies were obtained from Antibodies-

139 online (Germany), Horseradish peroxydase (type II), O-Phenylenediamine

140 dihydrochloride (OPD), $\mathrm{NaH}_{2} \mathrm{PO}_{4}$, Tween ${ }^{\circledR}$ 20, sodium acetate, sucrose, sodium azide,

$141 \mathrm{NaOH}, \mathrm{H}_{2} \mathrm{O}_{2}, \mathrm{BSA}$, (L)-Histidine and (D)-Arginine L-tyrosinamide, fluorescein and

142 Tris(hydroxymethyl) aminomethane were purchased from Sigma Aldrich (Saint-

143 Quentin, France). $\mathrm{NaCl}$ and $\mathrm{MgCl}_{2}$ were obtained from Chimie-Plus laboratoires

144 (Bruyères de Pouilly, France) and Panreac Quimica (Barcelona, Spain), respectively.

145 Chemicals were analytical grade and were used as received. DNA-Bind ${ }^{\mathrm{TM}}$ plates were

146 obtained from Corning (Netherlands) and Maxisorp ${ }^{\mathrm{TM}}$ plates were obtained from VWR

147 (France). Optical density of the reaction products was measured on a Tecan Infinite

148 M200 microplate reader (Lyon, France) at $492 \mathrm{~nm}$.

149 


\section{3. Method}

\section{$151 \quad 3.1$. Sample preparation}

152 Our Biochip models are small polymer containers, called micro-wells, where antibodies 153 samples are placed for the experiments. DNA-Bind ${ }^{\mathrm{TM}}$ plates were used for covalent 154 grafting (N-Oxysuccinimide functionalization allows random amine binding, Moreau et 155 al. 2011) whereas Maxisorp ${ }^{\mathrm{TM}}$ ones were used as sample containers for free antibodies.

156 The samples preparation was done following the same protocol as in Baque et al. 157 (2011a).

158 Briefly, antibodies were irradiated under two different states: grafted and free. All 159 samples were freeze-dried using the freeze-drying buffer described in Baqué et al. $160(2011 \mathrm{a}, \mathrm{b})$ and then sealed in a FoodSaver ${ }^{\mathrm{TM}}$ bag in dry atmosphere (silica gel was added 161 in the bag) and stored in the dark at $4^{\circ} \mathrm{C}$ before irradiation. All irradiation effects were 162 estimated on freeze-dried samples.

\section{3.2. Irradiation parameters}

\section{3.2.1. Conditioning of samples during irradiation}

166 In order to prevent potential degradations due to environmental changes (contact with 167 air, moisture, potential organic contaminants, etc.), all samples were irradiated under 168 their protecting packaging.

169 Micro-wells were irradiated directly in their sealed bags. The effect of the sealed bag, 170 considering its thickness and composition, was assessed using simulations performed 171 with the Geant4 toolkit (Agostinelli et al. 2003; Allison et al. 2006). We determined 172 that the influence of the bag during irradiations was negligible as very few particles 173 were stopped by this additional plastic layer and very few secondary particles were 174 created (data not shown). 


\section{3.2.2. Methodology adopted to choose irradiation parameters}

177 Numerical simulations give a basis to select the types of particles, energies and fluences 178 that we have to consider for irradiation experiments. However, this choice mainly 179 depends on technical constraints and the availability of irradiation facilities. As an

180 example, it is generally not possible to conduct ground-based experiments with the very

181 low flux of particles and the long duration of irradiation (months or years) encountered 182 in interplanetary space. In addition, due to analysis constraints (limit of detection, 183 uncertainties), it is also necessary to choose adequate irradiation parameters to ensure 184 that potential effects of particles on our targets will be measurable.

185 In the present study, when possible, we have chosen to use fluences in the same order of 186 magnitude as the surface density of grafted antibodies. The objective of our experiments 187 was to study the interaction between different types of particles and the antibody 188 molecule. Indeed, we wanted to determine if some particles could have a "direct effect" 189 on the recognition molecule: when a particle interacts with the molecule, is there 190 degradation or is the molecule completely insensitive to particle interaction? This approach can allow the identification of particles and energies more deleterious to antibodies (if existing) and the results obtained could help for studying the implementation of biochips on further exploration missions whatever the target object in

194 the Solar System. For instance, it could give precious data on the shielding design that 195 must be developed considering the expected irradiation environment.

197 To determine the density of antibodies grafted into a well, we used an innovative 198 quantification technique called ADECA (Coussot et al. 2011a; Coussot et al. 2011b; 199 Moreau et al. 2011) that was well adapted to our purpose. The grafting density of 
200 antibodies was defined around $8.8 \times 10^{11}$ antibodies $/ \mathrm{cm}^{2}$ with roughly $2.8 \times 10^{11}$

201 antibodies on the bottom and $5 \times 10^{11}$ antibodies on the sidewalls.

203 The fluence of particles reaching the antibodies was assessed using numerical

204 simulations performed with the Geant4 toolkit. Indeed, considering the geometry of the

205 well, it is obvious that antibodies grafted on the sides do not receive the same fluence of

206 particles as antibodies located at the bottom of the well. With a fluence of $3 \times 10^{12}$

207 particles $/ \mathrm{cm}^{2}$, the fluence of particles on the sidewalls was derived from the Geant4

208 simulations to be $2.4 \times 10^{-2}$ times the total fluence so $7.2 \times 10^{10}$ particles $/ \mathrm{cm}^{2}$.

209 Therefore, we can assess that $41 \%$ of antibodies grafted in a well have a significant

210 chance to interact with at least one particle. With this method, direct effects of particles

211 on antibodies can be detected if existing.

212 Lower fluences and higher fluences were also tested in some cases, with for example a

213 fluence of protons ten times lower $\left(3 \times 10^{11}\right.$ particles $\left./ \mathrm{cm}^{2}\right)$ or a fluence of neutrons ten

214 times higher $\left(3 \times 10^{13}\right.$ particles $\left./ \mathrm{cm}^{2}\right)$. In these cases, we estimate that $13 \%$ and $74 \%$ of

215 grafted antibodies interacted with a particle respectively.

216 Free antibody samples were prepared at a concentration of $15 \times 10^{16}$ antibodies/well.

217 The exact disposition of antibodies into the well is not defined but it is assumed that

218 they form several layers at the bottom of the well during freeze-drying. Therefore it is

219 not possible to determine the number of antibodies that could interact with incident

220 particles since each particle can penetrate in a column of piled antibodies.

\section{3.2.3. Neutron irradiation}

222 Neutron irradiation was performed at the cyclotron of Louvain-la-Neuve, in Belgium.

223 The high flux neutron irradiation facility uses a primary $50 \mathrm{MeV}$ deuteron beam on a 
224 beryllium target. The energy spectrum of the outcoming neutron beam is dominated by

225 a peak in the region of $23 \mathrm{MeV}$. The mean energy of neutrons is $16.56 \mathrm{MeV}$.

226 The current was set to $7 \mu \mathrm{A}$. Samples were positioned at two different distances so that

227 they received two different fluences. At a $12 \mathrm{~cm}$ distance, the fluence was $F_{H}=3 \times 10^{13}$

228 neutrons $/ \mathrm{cm}^{2}$ and the diameter of the beam was about $4.2 \mathrm{~cm}$ for $80 \%$ of homogeneity.

229 Whereas at a $40.5 \mathrm{~cm}$ distance, the fluence was $F_{L}=3 \times 10^{12}$ neutrons $/ \mathrm{cm}^{2}$ and the

230 diameter of the beam was about $10.2 \mathrm{~cm}$ for $80 \%$ of homogeneity. Samples were

231 irradiated during approximately 22 minutes.

\section{3.2.4. Proton irradiation}

233 Proton irradiation was also performed at the cyclotron of Louvain-La-Neuve, on the 234 Light Ion Facility (LIF) (Fig. 2 Top). This mono-energetic proton beam line can 235 produce up to $10^{9}$ protons $/ \mathrm{cm}^{2} / \mathrm{s}$ with energies from 10 to $75 \mathrm{MeV}$ (Berger et al. 1997).

236 The beam diameter is set to $10 \mathrm{~cm}$ and $\mathrm{a} \pm 10 \%$ of homogeneity is ensured.

237 Three irradiation campaigns took place between June 2010 and June 2012. Our samples 238 were irradiated with five different energies: $14.4 \mathrm{MeV}, 20.9 \mathrm{MeV}, 25.9 \mathrm{MeV}, 29.4 \mathrm{MeV}$ 239 and $50.5 \mathrm{MeV}$. The proton flux was set to $5 \times 10^{8}$ protons $/ \mathrm{cm}^{2} / \mathrm{s}$ so that the irradiations 240 lasted $1 \mathrm{~h} 40 \mathrm{~min}$ to reach the fluence of $3 \times 10^{12}$ protons $/ \mathrm{cm}^{2}$ for all the tested energies 241 and $10 \mathrm{~min}$ to reach $3 \times 10^{11}$ protons $/ \mathrm{cm}^{2}$ for $25.9 \mathrm{MeV}$ and $50.5 \mathrm{MeV}$.

\section{$242 \quad 3.2 .5$. Electron irradiation}

243 Electron irradiation was performed at the Institut Bergonié (Bordeaux, France) (Fig. 2 244 Bottom Left). The beam was calibrated to deliver $9 \mathrm{MeV}$ electrons and it was scanned 245 through a square collimator of $6 \mathrm{~cm}$ side. Samples were positioned at $1 \mathrm{~m}$ from the 246 source. The flux delivered by the facility was 200 MU (Monitor Unit) per minute with 1 247 MU corresponding to $5.38 \times 10^{6}$ electrons impacting the bottom of the well (Gobet et al. 
248 Submitted; unpublished data). Therefore, to deal with reasonable irradiation durations,

249 we decided to irradiate samples during 70 minutes corresponding to a fluence of $2.35 \mathrm{x}$

$25010^{11}$ electrons $/ \mathrm{cm}^{2}$.

251

\section{3.2.6. Carbon ions irradiation}

253 Carbon ions irradiation was performed at the LNS (Laboratori Nazionali del Sud)

254 facility of the INFN (Instituto Nazionale di Fisica Nucleare) in Catania. Samples were

255 presented vertically in front of the beam. A specific mask was designed to fix the ELISA

256 plate containing samples on a mobile device (Fig. 2 Bottom right) so that the whole

257 plate could be irradiated at once without any intervention in the irradiation room.

258 The beam was scanned through a square collimator of $17 \mathrm{~mm}$ side. Calibration for the

259 delivered dose has been done by means of a parallel plate ionization chamber.

260 Radiochromic films have been also used for minimizing gaps and overlaps between

261 irradiated areas in order to ensure a homogeneous irradiation of all samples.

262 The beam delivered ${ }^{12} \mathrm{C}$ ions with an energy of $62 \mathrm{MeV} /$ nuc. For this experiment, the

263 fluence applied was different from other experiments as it was not reasonable to reach 3

$264 \times 10^{12}$ carbon ions per $\mathrm{cm}^{2}$ in an adequate delay and safe conditions. Therefore, we

265 decided to study if energetic carbon ions could have an indirect effect on antibodies, i.e.

266 if those particles of such energy could interact with the sample environment so that it

267 could destabilize the whole system and degrade antibodies recognition performances.

268 The fluence was set to $2.16 \times 10^{6}$ particles $/ \mathrm{cm}^{2}$ and was determined using results

269 obtained with CREME 96 by Le Postollec et al. (2009a): it corresponds to the flux of

$270{ }^{12} \mathrm{C} 62 \mathrm{MeV} / \mathrm{nuc}$ ions at 1 A.U. (Astronomical Unit) delivered during 18 months

271 (representing an upper limit for a Mars mission). The irradiation of each square area 
272 lasted less than 20 seconds to reach the requested fluence so that the whole plate was

273 irradiated within about 15 minutes.

\section{3.3. Analysis protocol}

\section{3.3.1. Antibodies}

277 After irradiation, analyses were performed in order to define the irradiation effects on

278 the antibody performance. Protocols used here were detailed in previous studies (Baqué 279 et al. 2011a) and are summarized below.

281 Grafted antibodies were analyzed with a direct ELISA test (Baqué et al. 2011a). This

282 method, called A2HRP, focuses only on the recognition capability of the antibody's 283 antigen binding site (epitope) and does not give an insight on the degradation of the 284 entire antibody structure (Moreau et al. 2011). Briefly, the number of active antigen 285 binding sites was measured by quantifying the amount of antigen (HRP) specifically 286 retained by the antibodies. Indeed, the amount of HRP could be easily quantified using 287 external standards of free HRP as we have demonstrated that the enzymatic reactivity of 288 HRP was identical for free HRP, or HRP complexed to both free or grafted antibody 289 (Moreau et al. 2011).

291 Free antibodies were analyzed with a competitive ELISA test (Baqué et al. 2011a). 292 Briefly, in micro-well plates with freshly grafted anti-HRP antibodies, a defined amount 293 of HRP is placed in competition with diluted amounts of irradiated samples or controls. 294 After washing, the amount of HRP measured in the micro-well is inversely proportional 295 to the amount of active antibody in the sample. Based on competitive curves, we 296 calculated the half maximal inhibitory concentration (IC50). In our experiment, this 
297 concentration represents the amount of competitive antibody that should be added to

298 inhibit $50 \%$ of antigen binding to grafted antibodies. Between two competitive

299 experiments, both HRP and grafted antibody concentrations are maintained identical.

300 Thus IC50 values are influenced by the affinity of competitive antibodies for the HRP.

301 If the apparent affinity of competitive antibodies is reduced, then the IC50 measured

302 will increase.

303 3.3.2. Reference samples

304 To evaluate the possible irradiation effects on our samples, different references and 305 controls were prepared. Irradiation effect on antibody was evaluated by comparing 306 irradiated samples to non-irradiated controls (NIC). NIC were treated simultaneously

307 and in the same manner as the irradiated samples, though they were not submitted to 308 irradiation. In order to estimate the effects of transport, temperature cycles and light 309 exposure on biochip performances, reference samples were used. These reference 310 samples $\left(\mathrm{R} 4^{\circ} \mathrm{C}\right)$ were prepared at the same time as irradiated samples and NIC and were 311 stored in the laboratory at $4^{\circ} \mathrm{C}$ in the dark until analysis. As described by Baqué et al. 312 (2011a), all of the antibodies were freeze-dried using a specific buffer, which maintains 313 the anti-HRP antibody recognition capabilities after freeze-drying and during storage to 314 liquid reference levels. Results for grafted antibodies are therefore presented as 315 percentages of active antibodies for more clarity and in order to normalize all acquired 316 data during the several irradiation campaigns. This percentage is calculated by taking 317 the amount of HRP retained by NIC to $100 \%$. NIC and $\mathrm{R} 4{ }^{\circ} \mathrm{C}$ were confronted for each 318 campaign to reflect any damage caused by transport, handling etc.

\section{3.3.3. Statistical treatment}


320 Irradiation effects were evaluated by comparing the mean signal values obtained for

321 non-irradiated controls (NIC) and for irradiated samples. Thus, Student's t-tests were

322 used to compare irradiated samples distribution and references distribution, taking into

323 account the number of repetitions (from 4 to 18) and the standard deviation (SD) of

324 each distribution. The differences between these two distributions were considered

325 statistically significant with a $95 \%$ level of confidence when the calculated $p$-values

326 were below the 0.05 threshold value.

327

328 4. Results

329 4.1. Grafted antibodies

330 All the experiments performed on grafted antibodies are summarized in Table 1. This

331 table presents the type, energy and fluence of particles tested. It also specifies the

332 antibody grafting surface density allowing an assessment of the percentage of antibodies

333 receiving at least one particle for each tested fluence. The percentage of antibodies still

334 active after irradiation, calculated against non-irradiated controls (NIC), reveals possible

335 degradation induced only by radiation exposure. Indeed, the effects of transport

336 conditions are evaluated by confronting NIC with reference samples stored in the

337 laboratory $\left(\mathrm{R} 4^{\circ} \mathrm{C}\right)$, as described in paragraph 3.2.2. However, as for all the tested

338 conditions NIC proved to be significantly equal to $\mathrm{R} 4^{\circ} \mathrm{C}$ (not shown), only irradiation

339 effects are presented here.

340 Irradiation on the other hand had different effects on the tested antibodies. Indeed,

341 although no effect was detected with neutrons, electrons and ${ }^{12} \mathrm{C}$, significant effects

342 were observed with protons. Surprisingly, for high fluences, protons between 20 and 30

$343 \mathrm{MeV}$ significantly altered the antibody recognition performances, with losses around

$34430-35 \%$ and $p$-values between $10^{-4}$ and $10^{-8}$, but not at lower and higher energies.

345 Similarly, even at a lower fluence $25 \mathrm{MeV}$ protons produced a significant recognition 
346 loss, though limited to only $10-20 \%$, whereas at $50 \mathrm{MeV}$ no significant recognition loss

347 was recorded. In our model the antibody surface density was maintained identical for

348 the different exposure experiments therefore only $13 \%$ of antibodies should have

349 received at least one particle at the lowest proton fluence against $42 \%$ at the highest.

350 The protons' energy appears thus as a more damaging factor than the fluence, as only a

351 certain energy range (20-30 MeV) produced significant damage to antibodies regardless

352 of the fluence applied. However, by diminishing the ratio between the antibody surface

353 density and the particles' fluence by a factor $3(42 \%$ against $13 \%$ of antibodies

354 receiving at least one particle between high and low fluences respectively) the effect of

355 irradiation was greatly attenuated for $25 \mathrm{MeV}$ protons $(65 \%$ against $84 \%$ of active

356 antibodies respectively).

357 The other tested particles did not induce significant changes in antibody recognition 358 capabilities even at very high neutron fluence $\left(3 \times 10^{13}\right.$ particles $\left./ \mathrm{cm}^{2}\right)$ or with heavy 359 carbon ions at high energy $(62 \mathrm{MeV} / \mathrm{n})$.

\section{4.2. Free antibodies}

362 Free antibodies were irradiated by $25 \mathrm{MeV}$ and $50 \mathrm{MeV}$ protons and $17 \mathrm{MeV}$ neutrons 363 at different fluences $\left(3 \times 10^{11}\right.$ and $3 \times 10^{12}$ particles $/ \mathrm{cm}^{2}$ for protons and $3 \times 10^{12}$ and $3 \times$ $36410^{13}$ particles $/ \mathrm{cm}^{2}$ for neutrons). Results are summarized in Table 2. The irradiation 365 effect was estimated following the methodology described in Baqué et al. (2011a). 366 Briefly, when the half maximal inhibitory concentration (IC50) significantly increases, 367 it indicates that in average, the antibodies have lost recognition capabilities since HRP 368 has only one epitope to which the antibody binds (Moreau et al. 2011). 
370 No modification of free antibody recognition capabilities under proton irradiations at 50

$371 \mathrm{MeV}$ was observed. However, at $25 \mathrm{MeV}$, we highlight here a significant recognition

372 capability loss for free antibodies, leading to a significant increase in IC50 compared to

373 the NIC. The increase in IC50 value indicates that, in average, the antibody activity has

374 been deteriorated by $25 \mathrm{MeV}$ protons irradiation leading to partial or complete antigen

375 recognition site degradation. Based on a simplistic model, which considers that IC50

376 changes are only linked to a total loss of recognition capability, we can however

377 estimate the percentage of active antibodies compared to non-irradiated controls as

378 reported in Table 2. A maximum of 50\% of antibodies appear to have lost their 379 recognition capability when irradiated with a high fluence of $25 \mathrm{MeV}$ protons. Although

380 the other recorded changes in IC50 values after proton or neutron irradiation appear also

381 quite high, with 20 to $30 \%$ damaged antibodies (most notably after a high neutron

382 flux), they were not significantly different from the controls. These results however

383 point out a high variability in the samples, which can be problematic for repeatability

384 measurements of future space instruments.

\section{5. Discussion/Conclusion}

388 Based on Monte Carlo simulations of the radiation environment faced by a biochip 389 dedicated to explore Mars' surface (Le Postollec et al. 2009a), our team performed 390 several ground-based irradiation experiments on biochip recognition molecules. Even

391 though protons and neutrons clearly dominate the radiation spectrum during the Earth-

392 Mars transit and on the Martian surface, other particles might be equally deleterious to 393 biological molecules such as the antibodies used in biochips. Furthermore, a wide range 394 of particle energy and fluence can be considered according to the envisaged mission to 
395 Mars but also to other planetary bodies of interest in the Solar System. In the present 396 study, the irradiation effects of protons, neutrons, electrons and carbon ions on the 397 recognition capabilities of antibodies were therefore investigated at different energies 398 and fluences. Two antibodies formulations were submitted to irradiation in order to 399 broadly represent any future biochip-based space instruments as both grafted and free 400 antibodies are considered. Our experimental approach consisted of using particle 401 fluences in the same order of magnitude as grafted antibodies surface density in order to 402 measure any damaging effect occurring when a particle interacts with an antibody.

403 Among the tested particles, only protons significantly altered the antibodies recognition 404 capabilities. These damaging effects were however recorded only for a certain energy 405 range between 20 and $30 \mathrm{MeV}$ at both high and low fluences but confirmed for both 406 formulations (free and grafted antibodies). Indeed, at higher and lower protons energies 407 the antibodies recognition capabilities were not significantly altered. Irradiations of free 408 antibodies lead moreover to a high variability in the estimated recognition capabilities 409 of our antibodies samples.

410 Therefore, although the energy range of deleterious particles appears quite limited, a 411 biochip instrument performance would not be affected for a typical mission to Mars, as 412 the fluences of particles in this energy range will be significantly lower than the 413 antibody surface density. However, this result underlines that attention must be paid to 414 the ratio between antibody surface density and particles fluences expected for a given 415 mission. The biochip instrument must be designed so that antibody surface density is 416 much greater than incident protons fluence. Instrument shielding and/or antibodies 417 grafting density should be consequently adapted. 
419 In a similar ground-based study performed on five antibodies freeze-dried in different

420 protective molecular matrices, Derveni et al. (2012) pointed out the more damaging role

421 of processing and packaging than irradiation. Using doses of protons and neutrons at

422 high energies (50 and $47 \mathrm{MeV}$ respectively), comparable to the ones used in the present

423 work, they did not detect any evidence of activity loss due to irradiation for a typical

424 mission dose $\left(10^{11}\right.$ to $10^{12}$ protons $/ \mathrm{cm}^{2}$ and $10^{7}$ to $10^{8}$ neutrons $\left./ \mathrm{cm}^{2}\right)$. However, using

$42510^{13}$ protons $/ \mathrm{cm}^{2}$, most of the antibodies lost their activity. Thanks to these results they

426 suggested that further shielding or alternative radiation protection approaches would

427 need to be considered for long duration missions to other astrobiological targets. Our

428 present work confirms this suggestion. We propose that the ratio between the fluence of

429 protons and the surface density of antibodies has to be much lower than unity to prevent

430 important loss of activity.

432 The main limitation of ground-based studies is that each constraint is generally studied 433 individually and for a limited period of time that is not representative of a real space 434 mission. In particular, the effect of cosmic rays is generally studied at a given energy (or 435 a limited range of energies) and for one type of particle in a single experiment.

436 Moreover, additional constraints and hazards are expected for a space instrument. Long 437 term storage, temperature variations, contamination risks, launch, landing and 438 transportations vibrations and shocks should all be taken into account in the design and 439 testing of a space dedicated instrument. For these reasons, a real space exposure of 440 biochip prototypes has been attempted in the past by the LMC team for a short-term 441 mission aboard the BIOPAN platform on a Russian Foton spacecraft (Derveni et al. 442 2013) and ground-based and field studies have been performed for the SOLID prototype 443 (Parro et al. 2008; Sobrado et al. 2014). Furthermore, in the frame of the BiOMAS 
444 project, biochip samples are currently exposed to real space conditions inside the

445 EXPOSE-R2 platform of ESA, part of the Photochemistry on the Space Station (PSS)

446 project, which was installed on the outside of the Zvezda module of the International

447 Space Station (ISS) in August 2014 (Vigier et al. 2013).

448 The long-duration exposure of the EXPOSE missions (Rabbow et al. 2009, 2012, 2015)

449 range from 12 to 18 months in the LEO environment of the ISS. The radiation

450 environment at this altitude, although not equivalent to interplanetary space or the

451 Martian surface, will allow anyway for a much better estimate of the long-term

452 resistance of immunoassays instruments for space applications.

453 Nevertheless, due to the high number of potentially hazardous factors encountered

454 during a space mission, ground-based studies are essential to isolate the most damaging

455 ones and thus propose adequate shielding or handling procedures.

457 Thus, our results from ground-based irradiation campaigns globally indicate that cosmic 458 rays might not alter the final performance of a biochip-based instrument in a typical 459 Martian mission, when antibodies are used as binders to detect the presence or the 460 absence of a target compound. The damaging effects of 20-30 MeV protons recorded in 461 the present study should not however be overlooked and further testing on-ground will 462 be necessary to support and interpret data from real space exposure missions.

\section{Acknowledgments}

466 The research leading to these results has received funding from the European Union

467 Seventh Framework Programme FP7/2007-2013 under Grant Agreement no 262010 468 ENSAR. 
469 We would like to thank the French National Space Agency (CNES) (Convention 470 number DCT/SI/IM/2009-17733) and the Interdisciplinary CNRS program

471 "Environnements Planétaires et Origines de la Vie" for financial support.

472 We also thank the Louvain-la-Neuve cyclotron facility staff, the Institut Bergonié staff 473 and the staff from the Laboratori Nazionali del Sud of the Instituto Nazionale di Fisica 474 Nucleare for their help during irradiation experiments.

475 


\section{References}

Agostinelli, S. et al. (2003) Geant4-a simulation toolkit. Nucl. Instrum. Methods Phys. Res. Sect. Accel. Spectrometers Detect. Assoc. Equip.. 506(3), 250-303.

Allison, J. et al. (2006) Geant4 developments and applications. IEEE Trans. Nucl. Sci.. 53(1), 270-278.

Baqué, M. et al. (2011a) Investigation of Low-Energy Proton Effects on Aptamer Performance for Astrobiological Applications. Astrobiology. 11(3), 207-211.

Baqué, M., Le Postollec, A., Coussot, G., Moreau, T., Desvignes, I., Incerti, S., Moretto, P., Dobrijevic, M., \& Vandenabeele-Trambouze, O. (2011b) Biochip for astrobiological applications: Investigation of low energy protons effects on antibody performances. Planet. Space Sci.. 59(13), 1490 - 1497.

Berger, G., Ryckewaert, G., Harboe-Sorensen, R., \& Adams, L. (1997) Cyclone-A multipurpose heavy ion, proton and neutron see test site. In 1997 RADECS Conference Data Workshop. p. 51.

Coussot, G., Faye, C., Ibrahim, A., Ramonda, M., Dobrijevic, M., Postollec, A., Granier, F., \& Vandenabeele-Trambouze, O. (2011) Aminated dendritic surfaces characterization: a rapid and versatile colorimetric assay for estimating the amine density and coating stability. Anal. Bioanal. Chem.. 399(6), 22952302.

Coussot, G., Perrin, C., Moreau, T., Dobrijevic, M., Postollec, A.L., \& VandenabeeleTrambouze, O. (2011) A rapid and reversible colorimetric assay for the characterization of aminated solid surfaces. Anal. Bioanal. Chem.. 399(3), 10611069.

Derveni, M., Allen, M., Sawakuchi, G.O., Yukihara, E.G., Richter, L., Sims, M.R., \& Cullen, D.C. (2013) Survivability of Immunoassay Reagents Exposed to the Space Radiation Environment on board the ESA BIOPAN-6 Platform as a Prelude to Performing Immunoassays on Mars. Astrobiology, 130103060450009.

Derveni, M., Hands, A., Allen, M., Sims, M.R., \& Cullen, D.C. (2012) Effects of Simulated Space Radiation on Immunoassay Components for Life-Detection Experiments in Planetary Exploration Missions. Astrobiology. 12(8), 718-729.

de Diego-Castilla, G., Cruz-Gil, P., Mateo-Martí, E., Fernández-Calvo, P., Rivas, L.A., \& Parro, V. (2011) Assessing Antibody Microarrays for Space Missions: Effect of Long-Term Storage, Gamma Radiation, and Temperature Shifts on Printed and Fluorescently Labeled Antibodies. Astrobiology. 11(8), 759-773.

Gobet, F. et al. (Submitted) Experimental and Monte Carlo absolute characterization of a medical electron beam. Radiat. Meas. 
Hassler, D.M. et al. (2013) Mars' Surface Radiation Environment Measured with the Mars Science Laboratory's Curiosity Rover. Science, 1244797.

Kim, M.-H.Y. et al. (2014) Comparison of Martian Surface Radiation Predictions to the Measurements of Mars Science Laboratory Radiation Assessment Detector (MSL/RAD). In American Geophysical Union Fall 2014 Meeting. San Francisco, CA, United States.

Manchado, J.M., Sebastián, E., Romeral, J., Sobrado-Vallecillo, J., Herrero, P.L., Compostizo, C., Gómez-Elvira, J., \& Parro, V. (2015) SOLID SPU: A TRL 5-6 Sample Preparation Instrument for Wet Chemistry Analysis on Mars. In Lunar and Planetary Science Conference. p. 1222.

Martins, Z. (2011) In situ biomarkers and the Life Marker Chip. Astron. Geophys.. 52(1), 1.34-1.35.

McKay, C.P. et al. (2013) The Icebreaker Life Mission to Mars: A Search for Biomolecular Evidence for Life. Astrobiology. 13(4), 334-353.

McKay, D.S., Steele, A., Allen, C., Thomas-Keptra, K., Schweitzer, M.H., Priscu, J., Sears, J., Avci, R., \& Firman, K. (2000) Mars immunoassay life detection instrument (MILDI). Lunar Planet Inst Contrib. (1062 Part 2), 219-220.

McKenna-Lawlor, S., Gonçalves, P., Keating, A., Reitz, G., \& Matthiä, D. (2012) Overview of energetic particle hazards during prospective manned missions to Mars. Planet. Space Sci.. 63-64(0), 123-132.

Moreau, T., Faye, C., Baqué, M., Desvignes, I., Coussot, G., Pascal, R., \& Vandenabeele-Trambouze, O. (2011) Antibody-based surfaces: Rapid characterization using two complementary colorimetric assays. Anal. Chim. Acta. 706(2), 354-360.

Parro, V. et al. (2005) Instrument development to search for biomarkers on mars: Terrestrial acidophile, iron-powered chemolithoautotrophic communities as model systems. Planet. Space Sci. 53(7), 729-737.

Parro, V. et al. (2008) SOLID2: an antibody array-based Life-detector instrument in a Mars drilling simulation experiment (MARTE). Astrobiology. 8(5), 987-999.

Parro, V. et al. (2011a) Classification of Modern and Old Rio Tinto Sedimentary Deposits Through the Biomolecular Record Using a Life Marker Biochip: Implications for Detecting Life on Mars. Astrobiology. 11(1), 29-44.

Parro, V. et al. (2011b) SOLID3: a multiplex antibody microarray-based optical sensor instrument for in situ Life detection in planetary exploration. Astrobiology. 11(1), 15-28.

Le Postollec, A. et al. (2007) Development of a Biochip dedicated to planetary exploration. First step: resistance studies to space conditions. In Journées $S F 2 A$ 2007 Semaine de l'Astrophysique Française 2007. 
Le Postollec, A. et al. (2009a) Monte Carlo Simulation of the Radiation Environment Encountered by a Biochip During a Space Mission to Mars. Astrobiology. 9(3), $311-323$.

Le Postollec, A., Coussot, G., Baqué, M., Incerti, S., Desvignes, I., Moretto, P., Dobrijevic, M., \& Vandenabeele-Trambouze, O. (2009b) Investigation of Neutron Radiation Effects on Polyclonal Antibodies (IgG) and Fluorescein Dye for Astrobiological Applications. Astrobiology. 9(7), 637-645.

Rabbow, E. et al. (2009) EXPOSE, an astrobiological exposure facility on the International Space Station - from proposal to flight. Orig. Life Evol. Biospheres. 39(6), 581-598.

Rabbow, E. et al. (2012) EXPOSE-E: an ESA astrobiology mission 1.5 years in space. Astrobiology. 12(5), 374-386.

Rabbow, E. et al. (2015) The astrobiological mission EXPOSE-R on board of the International Space Station. Int. J. Astrobiol..14(1), 3-16.

Sephton, M.A., Sims, M.R., Court, R.W., Luong, D., \& Cullen, D.C. (2013) Searching for biomolecules on Mars: Considerations for operation of a life marker chip instrument. Planet. Space Sci.. 86, 66-74.

Sims, M., Cullen, D., Bannister, N., Grant, W., Henry, O., Jones, R., McKnight, D., Thompson, D.P., \& Wilson, P. (2005) The specific molecular identification of life experiment (SMILE). Planet. Space Sci.. 53(8), 781-791.

Sims, M.R. et al. (2012) Development status of the life marker chip instrument for ExoMars. Planet. Space Sci.. 72(1), 129-137.

Smith, H. \& Parro, V. (2014) Planetary Protection Plan for an Antibody based instrument proposed for Mars2020. In 40th COSPAR Scientific Assembly. p. 3140.

Sobrado, J.M., Martín-Soler, J., \& Martín-Gago, J.A. (2014) Mimicking Mars: A vacuum simulation chamber for testing environmental instrumentation for Mars exploration. Rev. Sci. Instrum. 85(3), 035111.

Vigier, F. et al. (2013) Preparation of the Biochip experiment on the EXPOSE-R2 mission outside the International Space Station. Adv. Space Res. 52(12), 21682179.

Wang, J. (2006) Electrochemical biosensors: Towards point-of-care cancer diagnostics. Biosens. Bioelectron.. 21(10), 1887-1892.

Zeitlin, C. et al. (2013) Measurements of Energetic Particle Radiation in Transit to Mars on the Mars Science Laboratory. Science. 340(6136), 1080-1084. 


\section{$589 \quad$ Figure legends}

590 Fig. 1: Simulated spectra of particle fluxes, as a function of energy, during the Earth-

591 Mars transit (left) and at Mars' surface (right) with the energy range of particles

592 investigated in this study (red zone). This figure is an adaptation of Fig. 8 in Le

593 Postollec et al. (2009a).

594 Fig. 2: Top: Proton irradiation using the Light Ion Facility (LIF) at the cyclotron of

595 Louvain-la-Neuve. The source is located on the left in this picture and the samples are

596 placed on the right behind a metal slide with a $10 \mathrm{~cm}$ diameter hole. Several removable

597 disks are placed between the source and the samples to allow the modulation of protons

598 energy. Bottom Left: Picture of the facility at the Institut Bergonié where samples were

599 irradiated with $9 \mathrm{MeV}$ electrons. Bottom Right: Mobile device developed to ensure the

600 ELISA plate motion during carbon ions irradiation at LNS (Catania). 
SIX MONTHS

EARTH-MARS TRAVEL

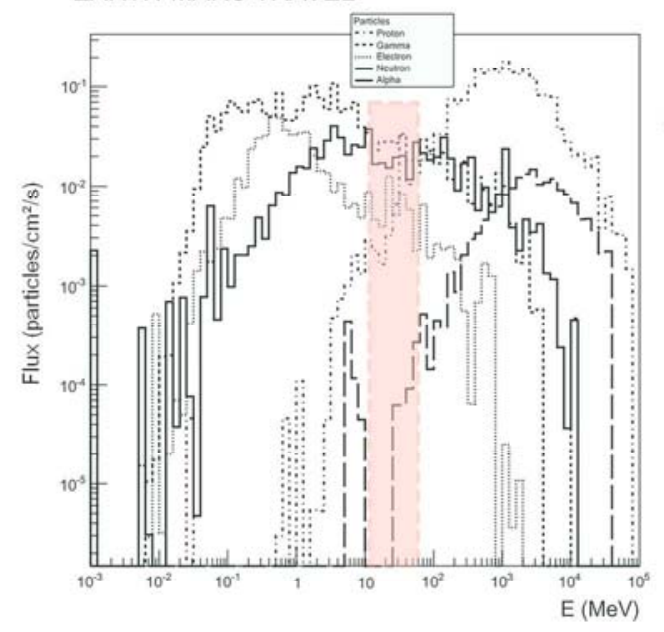

ONE MONTH MARS STAY

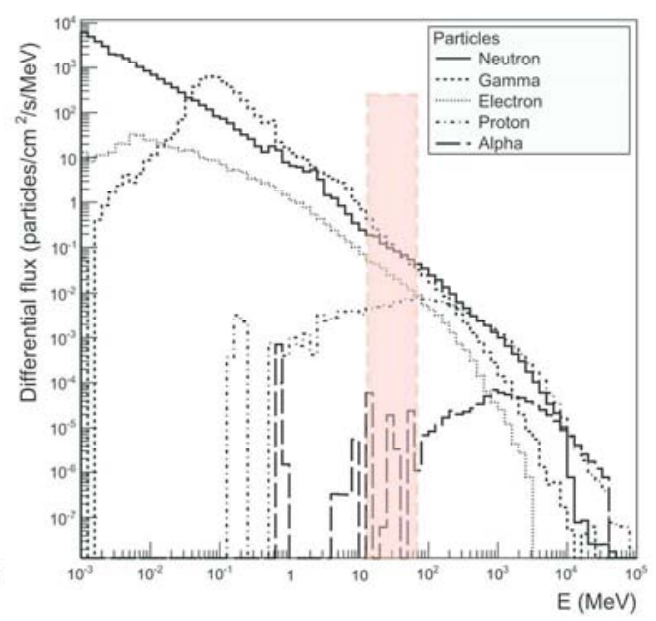

Tested particles and energies

Proton: $15,20,25,30,50 \mathrm{MeV}$

Neutron: $17 \mathrm{MeV}$

Electron: $9 \mathrm{MeV}$

Carbon: $62 \mathrm{MeV} / \mathrm{n}$

$143 \times 98 \mathrm{~mm}(300 \times 300$ DPI $)$ 


\section{Page 27 of 29}
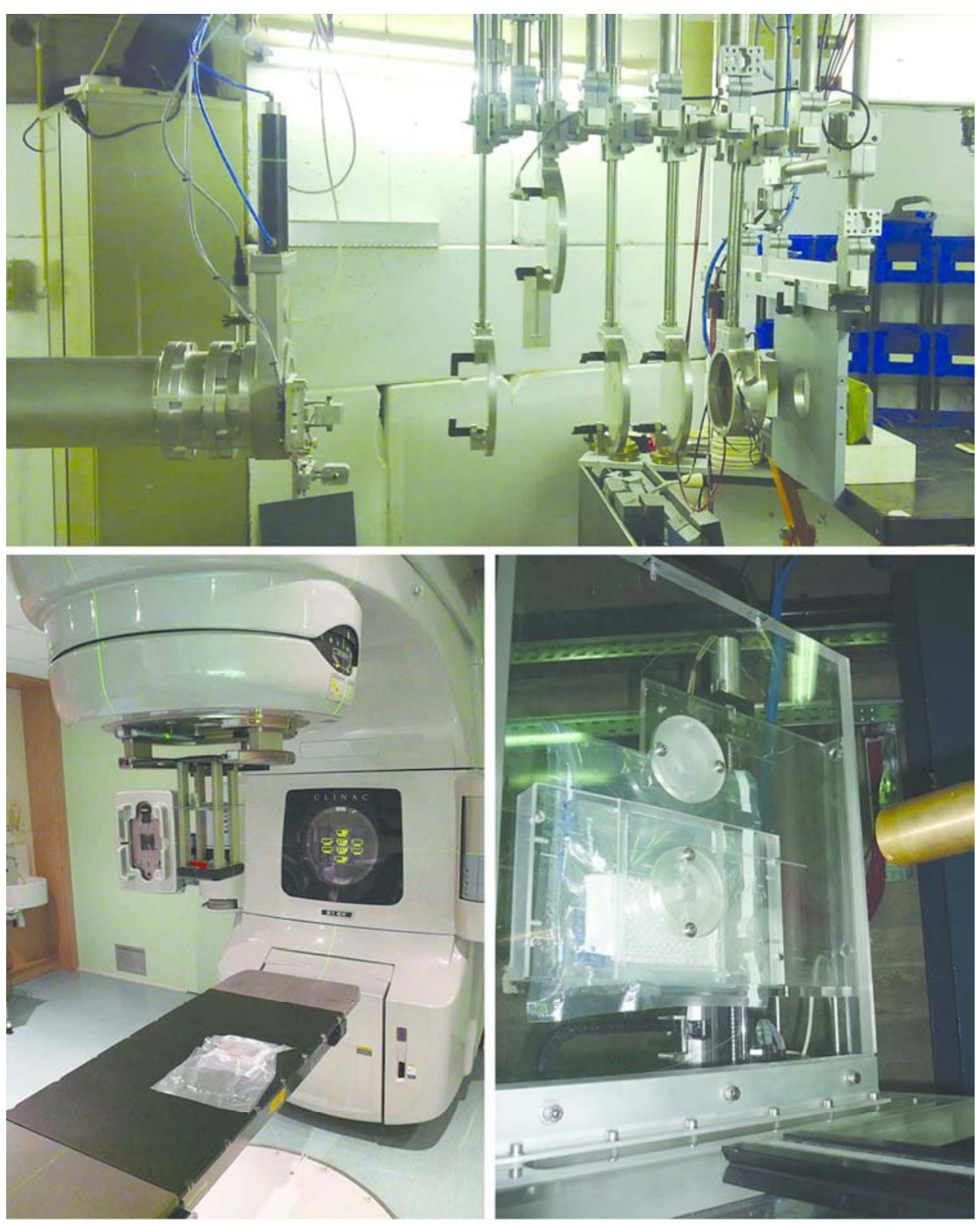

$99 \times 124 \mathrm{~mm}(300 \times 300 \mathrm{DPI})$ 
Table 1. Influence of neutron, proton, electron and carbon radiation effects on grafted antibodies recognition capability at different fluences. The percentages of active antibodies were normalized using the NIC that were thus fixed at $100 \%$. The percentage of antibodies receiving at least one particle was calculated according to the antibody surface density, the tested fluence and the sample geometry. SD, standard deviation; $n$ is the number of measurements. $p$-value $<0.05$ (in bold) indicate samples that are different to NIC at $95 \%$ of confidence.

\begin{tabular}{|c|c|c|c|c|c|c|c|c|c|c|c|}
\hline \multirow{3}{*}{$\begin{array}{c}\begin{array}{c}\text { Fluence } \\
\text { particles } / \mathrm{cm}^{2}\end{array} \\
\text { Energy } \mathrm{MeV}\end{array}$} & \multicolumn{7}{|c|}{ Protons } & \multicolumn{2}{|c|}{ Neutrons } & Electrons & ${ }^{12} \mathrm{C}$ \\
\hline & \multicolumn{2}{|c|}{$3 \times 10^{11}$} & \multicolumn{6}{|c|}{$3 \times 10^{12}$} & $3 \times 10^{13}$ & $2.3 \times 10^{11}$ & $2.2 \times 10^{6}$ \\
\hline & 25 & 50 & 15 & 20 & 25 & 30 & 50 & 17 (me & nergy) & 9 & $62 \mathrm{MeV} / \mathrm{n}$ \\
\hline $\begin{array}{c}\text { Antibodies } \\
\text { receiving at least } 1 \\
\text { particle } \% *\end{array}$ & \multicolumn{2}{|c|}{13} & \multicolumn{6}{|c|}{41} & 88 & 10 & $<1$ \\
\hline $\begin{array}{l}\text { Percentage of } \\
\text { active antibodies } \\
\% \pm \operatorname{SD}(n)\end{array}$ & $\begin{array}{c}84 \pm 9 \\
(13)\end{array}$ & $\begin{array}{c}89 \pm 10 \\
(5)\end{array}$ & $\begin{array}{c}97 \pm 19 \\
(15)\end{array}$ & $\begin{array}{c}62 \pm 7 \\
(5)\end{array}$ & $\begin{array}{c}65 \pm 12 \\
(13)\end{array}$ & $\begin{array}{c}73 \pm 8 \\
(5)\end{array}$ & $\begin{array}{l}92 \pm 14 \\
(5)\end{array}$ & $\begin{array}{c}100 \pm 7 \\
(4)\end{array}$ & $\begin{array}{c}96 \pm 4 \\
(5)\end{array}$ & $\begin{array}{c}98 \pm 4 \\
(4)\end{array}$ & $\begin{array}{c}102 \pm 10 \\
(10)\end{array}$ \\
\hline$p$-value & $6.98 \times 10^{-4}$ & 0.083 & 0.612 & $4.7 \times 10^{-4}$ & $3.95 \times 10^{-8}$ & $6.47 \times 10^{-4}$ & 0.283 & 0.938 & 0.136 & 0.656 & 0.839 \\
\hline
\end{tabular}

* Antibody surface density is equal to $8.8 \times 10^{11} \mathrm{Ab} / \mathrm{cm}^{2}$ for all experiments. 
Table 2. Influence of neutron and proton irradiation on free-antibody recognition capability at different fluences. IC50 $(\mu \mathrm{g} / \mathrm{mL})$, half maximal inhibitory concentration; SD, standard deviation; $n$ is the number of measurements. The percentages of active antibodies were estimated in comparison with NIC. $p$-values $<0.05$ (in bold) indicate samples that are different to non-irradiated controls at $95 \%$ of confidence.

\begin{tabular}{|c|c|c|c|c|c|c|c|}
\hline \multirow{4}{*}{$\begin{array}{c}\text { Fluence particles } / \mathrm{cm}^{2} \\
\text { Energy MeV } \\
\text { IC } 50(\mu \mathrm{g} / \mathrm{mL}) \pm \mathrm{SD}(\mathrm{n})\end{array}$} & \multicolumn{4}{|c|}{ Protons } & \multicolumn{2}{|c|}{ Neutrons } & \multirow{3}{*}{$\begin{array}{l}\text { Non- } \\
\text { irradiated } \\
\text { controls } \\
\text { (NIC) }\end{array}$} \\
\hline & \multicolumn{2}{|c|}{$3 \times 10^{11}$} & \multicolumn{3}{|c|}{$3 \times 10^{12}$} & $3 \times 10^{13}$ & \\
\hline & 25 & 50 & 25 & 50 & \multicolumn{2}{|c|}{17 (mean energy) } & \\
\hline & $\begin{array}{l}3.1 \pm 0.2 \\
\quad(4)\end{array}$ & $\begin{array}{l}4.1 \pm 1.0 \\
\text { (7) }\end{array}$ & $\begin{array}{l}4.7 \pm 1.0 \\
\quad(7)\end{array}$ & $\begin{array}{c}3.8 \pm 0.8 \\
(8)\end{array}$ & $\begin{array}{l}3.2 \pm 0.2 \\
\quad(4)\end{array}$ & $\begin{array}{l}4.2 \pm 1.4 \\
\quad(8)\end{array}$ & $\begin{array}{l}3.2 \pm 0.6 \\
\quad(18)\end{array}$ \\
\hline $\begin{array}{c}\text { Percentage of active } \\
\text { antibodies } \%\end{array}$ & 100 & 71 & 50 & 79 & 105 & 73 & 100 \\
\hline$p$-value & 0.946 & 0.059 & 0.004 & 0.085 & 0.666 & 0.163 & \\
\hline
\end{tabular}

